\title{
Long non coding RNA OIP5-AS1 promotes metastasis of breast cancer via miR-340-5p/ZEB2 axis
}

\author{
LINGJUN MENG $^{1}$, XIAOJING YUE ${ }^{2}$, DI ZHOU ${ }^{1}$ and HONGJUN LI ${ }^{3}$ \\ ${ }^{1}$ Department of Hematology and Oncology, China-Japan Union Hospital Affiliated to Jilin University, Changchun, \\ Jilin 130033; ${ }^{2}$ Department of Developmental and Behavioral Pediatrics, The First Hospital of Jilin University, \\ Changchun 130021; ${ }^{3}$ Health Management Medical Center, China-Japan Union Hospital Affiliated to Jilin University, \\ Changchun, Jilin 130033, P.R. China
}

Received January 22, 2020; Accepted July 20, 2020

DOI: $10.3892 /$ or.2020.7724

\begin{abstract}
Breast cancer is the most common invasive cancer in women with the highest number of related deaths which is caused by distal metastasis. Recently, integrated analysis of gene expression profile suggested widespread gene dysregulation in various types of cancer. Research in the past decade has focused on long non-coding RNAs (IncRNAs), particularly in cell proliferation, tumor progression and metastasis. OPA-interacting protein 5 antisense transcript 1 (OIP5-AS1) is an evolutionarily conserved long non-coding RNA that has been linked to oncogenesis in multiple cancers. In breast cancer, dysregulation of OIP5-AS1 was reported but the precise role in cancer development and progression remains unclear. In the present study, using small interfering RNA (siRNA) targeting OIP5-AS1, it was shown that knockdown of OIP5-AS1 was associated with alteration of EMT markers and suppressed migration and invasion of breast cancer cells. Among the EMT-related transcription factors, ZEB1 and ZEB2 were significantly downregulated with OIP5-AS1 knockdown. Computational analysis and a dual-luciferase reporter system identified miR-340-5p was the target gene for OIP5-AS1. Further experiments verified the function of OIP5-AS1 in cell invasion was dependent on miR-340a-5p through regulating target gene ZEB2. In vivo study demonstrated that overexpressing OIP5-AS1 in breast cancer cells promoted lung metastasis in nude mice. The findings of the present study revealed the mechanism of OIP5-AS1 in breast cancer metastasis. Overall, our study may provide a potential therapeutic target for breast cancer metastasis.
\end{abstract}

Correspondence to: Professor Hongjun Li, Health Management Medical Center, China-Japan Union Hospital Affiliated to Jilin University, 126 Xiantai Street, Changchun, Jilin 130033, P.R. China

E-mail: hj_li@jlu.edu.cn

Key words: OIP5-AS1, miR-340-5p, ZEB2, breast cancer, metastasis

\section{Introduction}

Currently, breast cancer remains a leading health problem and constitutes one of the most severe burdensome diseases in females around the world despite understanding of underlying molecular mechanisms (1). Tumor metastasis is diagnosed in approximately $30 \%$ of breast cancer patients and is the major cause of cancer-related deaths (2). The prognosis for most patients with metastatic breast cancer is unfavorable with a median overall survival range from 2 to 3 years (3). Generally, breast cancer can be categorized into four subtypes [luminal A, luminal B, human epidermal growth factor receptor 2 (HER2) positive and triple negative], which are defined using immunohistochemical breast tumor markers (4). These four subtypes have the potential risk of distant metastasis but with differential site-specific metastatic patterns (5). Currently, breast cancer metastasis from primary tumor to distant organs occurs through a sequential molecular cascade including local angiogenesis for tumor growth, invasion of the surrounding tissue, intravasation of the carcinoma cells into the blood or lymphatic vessels, dissemination and proliferation at secondary neoplastic foci (6). These carcinoma cells obtain mesenchymal features and suppress their epithelial features through the epithelial-to-mesenchymal transition (EMT) process to promote an invasive and metastatic phenotype (7). Multiple transcription factors coordinate EMT programs. Among them, zinc-finger E-box-binding (ZEB) transcription factors, ZEB1 and ZEB2, are two EMT regulators that either repress or activate transcription in various types of cancer (8). Furthermore, ZEB2 was reported to negatively correlate with the epithelial marker E-cadherin in breast cancer cells involved in breast cancer progression (9).

Long non-coding RNAs (lncRNAs) are a class of transcripts containing more than 200 nucleotides in length with limited protein-coding capacity (10). Recent findings have shown that dysregulation of lncRNAs is involved in cell proliferation, tumor progression and metastasis in cancers (11). Functionally, lncRNAs interact with proteins and other RNAs to regulate their activities and cellular location. Furthermore, lncRNAs act as molecular sponges for miRNAs that block the binding activity for target transcripts (12). In breast cancer, several lncRNAs have been identified as either oncogenic 
or tumor suppressive factors, such as X-inactive-specifictranscript (XIST), HOX antisense intergenic RNA (HOTAIR), growth arrest specific 5 (GAS5) and metastasis-associated lung adenocarcinoma transcript 1 (MALAT1) (13-16). A systematic analysis of the correlation has been carried out between these dysregulated lncRNAs and breast cancer clinicopathology and survival suggesting a pivotal role in cancer development (17). Increasing lncRNAs have been shown to participate in specific cancer types but more often exert general function in a broad spectrum of cancer.

OPA-interacting protein 5 antisense transcript 1 (OIP5-AS1) is an evolutionarily conserved long non-coding RNA that is transcribed from opposite direction to the OIP5 gene. It was first shown to be expressed in the nervous system and was essential for neurogenesis during embryonic development (18). The functions of OIP5-AS1 in multiple human cancers have been reported to be associated with oncogenesis $(19,20)$. In breast cancer, OIP5-AS1 levels are upregulated in breast tumor tissue and correlated with tumor size, metastatic status of lymph nodes, pathological grading and TNM stage (21).

In the present study, we investigated the role of OIP5-AS1 in breast cancer metastasis using the in vitro and in vivo models showing that OIP5-AS1 regulates ZEB2 expression by acting as ceRNA for miR-340-5p.

\section{Materials and methods}

Cell culture and transfection. Breast cancer cell lines MCF-7, MDA-MB-231, ZR-75, MDA-MB-468, SKBR3 and normal human epithelial cell line MCF-10A were purchased from the American Type Culture Collection (ATCC). MCF-10A cells were cultured in MEBM (Lonza) and supplemented with $100 \mathrm{ng} / \mathrm{ml}$ cholera toxin. ZR-75 and SKBR3 cell lines were maintained in RPMI-1640 medium (Sigma) containing 10\% FBS, 2 mM L-glutamine and $2 \%$ penicillin and streptomycin. MCF-7, MDA-MB-231, and MDA-MB-468 cell lines were maintained in DMEM supplemented with $10 \%$ FBS plus $2 \%$ penicillin and streptomycin. Cells were cultured in a humidified incubator at $37^{\circ} \mathrm{C}$ with $5 \% \mathrm{CO}_{2}$.

The pre-designed siOIP5-AS1 and siZEB2 were purchased from ThermoFisher (no. 4390771, no. AM16708) and transfected into cells using Lipofectamine RNAiMAX reagent (ThermoFisher) following the manufacturer's instructions. miR-340-5p mimics, negative control mimics, miR-340-5p inhibitors and negative control inhibitors were purchased from GenePharma and transfected into cells using Lipofectamine 2000 reagent (ThermoFisher), according to the manufacturers' instructions. miR-340-5p mimic: 5'-UUAUAA AGCAAUGAGACUGAUU-3' and miR-340-5p inhibitor: 5'-AAUCAGUCUCAUUGCUUUAUAA-3'. Cells were used for further experiments at $48 \mathrm{~h}$ after transfection.

Wound healing assay. MCF-7 and MDA-MB-231 cells were seeded in 12-well plates and transfected with either siNC or siOIP5-AS1. A linear wound was scratched across the center of the well using a sterile pipette tip. The images of wound closure were captured after $24 \mathrm{~h}$ using Olympus microscope (x10).

Transwell invasion assay. The invasion of MCF-7 and MDA-MB-231 cells was detected using matrigel-coated or non-coated chambers with a pore size of $0.8 \mu \mathrm{M}$. The transfected cells were seeded into the upper chamber in DMEM with $1 \%$ FBS and the lower chamber was filled with $10 \%$ FBS as a chemoattractant. After 24-h incubation in the humidified incubator at $37^{\circ} \mathrm{C}$ with $5 \% \mathrm{CO}_{2}$, cells in the upper chamber were removed and cells in the lower side were fixed with $4 \%$ PFA and stained by $1 \%$ crystal violet. Stained cells were then visualized and imaged at a $\times 20$ magnification by a light microscope.

Western blot analysis. The cellular proteins were extracted in RIPA buffer supplemented with protease inhibitor. The protein concentration was quantified using BCA protein assay and $20 \mu \mathrm{g}$ of each protein sample was loaded and analyzed by $10 \%$ sodium dodecyl sulfate (SDS)-polyacrylamide gel electrophoresis (PAGE) system. Then, proteins were transferred to a PVDF membrane. The membrane was blocked in 5\% BSA and probed with primary antibodies: Anti-ZEB2 (1:1,000; no. ab138222, Abcam), anti-E-cadherin (1:1,000; no. 14472, Cell signaling), anti-N-cadherin (1:1,000; no. ab18203, Abcam), anti-vimentin (1:1,000; no. ab92547, Abcam), anti-ZEB1 (1:1,000; no. 70512, Cell signaling), anti-Snail (1:1,000; no. IMG-6639A, Novus Biologicals), anti-Slug (1:1,000; no. 9585, Cell signaling), anti-Twist (1:1,000; no. 69366, Cell signaling) and anti-GAPDH (1:2,000; no. ab8245, Abcam). Then, the membrane was incubated with peroxidase-conjugated anti-mouse or anti-rabbit secondary antibody (1:2,000, nos. NEF822001EA; NEF812001EA, PerkinElmer). Immunoreactivity bands were detected by chemiluminescence and the intensity of the bands was quantified using Image Lab Software (Bio Rad, China).

RNA fluorescence in situ hybridization (FISH). A Cy3-labeled set of probes recognizing OIP5-AS1 was designed and synthesized by Biosearch Technologies. The MCF-7 cells were cultured on coverslips for $24 \mathrm{~h}$ and then fixed in $4 \%$ PFA. After permeabilization with $70 \%$ ethanol at $4{ }^{\circ} \mathrm{C}$ for $1 \mathrm{~h}$, cells were hybridized with the OIP5-AS1 probes dissolved in hybridization buffer (no. SMF-HB1-10, Biosearch Technologies) at $37^{\circ} \mathrm{C}$ in the dark for $16 \mathrm{~h}$. The nucleus was stained with DAPI. Images were captured using a confocal microscope (Olympus).

Quantitative real-time PCR (RT-PCR). Total RNA was extracted from cells (MCF-7, MDA-MB-231, ZR-75, MDA-MB-468, SKBR3, and MCF-10A) using TRIzol ${ }^{\circledR}$ reagent (Thermo Fisher). Total RNA $(1 \mu \mathrm{g})$ was reverse transcribed into cDNA and a SYBR-Green quantitative real-time PCR Master Mix kit was used to detect qPCR signals. The targeted gene expression was normalized with $G A P D H$ and calculated using $2^{-\Delta \Delta \mathrm{Cq}}$ method (22). The primer sequences used were: OIP5-AS1: 5'-TGCAACCCAAGGTGGATACT-3' and 5'-GAGAGACTGCAGTGAGCAGA-3'; ZEB2: 5'-CAGCTC TTCCACCTCAAAGC-3' and 5'-TCCTTGTTTCCGCTG GTACT-3'; GAPDH: 5'-GTCGGAGTCAACGGATTTGG-3' and 5'-TGACGGTGCCATGGAATTTG-3'. For the detection of miR-340-5p, stem-loop qRT-PCR was performed using miScript SYBR-Green PCR Kit with U6 small nuclear RNA as an internal control (Qiagen). The following thermocycling conditions were used in the experiments: PCR initial activation at $95^{\circ} \mathrm{C}$ for $15 \mathrm{~min}$, followed by 40 cycles of denaturation 
at $94^{\circ} \mathrm{C}$ for $15 \mathrm{sec}$, annealing at $55^{\circ} \mathrm{C}$ for $30 \mathrm{sec}$ and an extension at $70^{\circ} \mathrm{C}$ for $30 \mathrm{sec}$.

RNA immunoprecipitation assay. Magna RIP kit (Millipore) was used for RNA immunoprecipitation experiments. The procedure was performed following the manufacturer's protocol. Briefly, after miR-340-5p mimics or NC mimics transfection, the cells were lysed in RIP lysis buffer. The cell lysate was incubated with either Ago2 antibody or control IgG together with protein $\mathrm{A} / \mathrm{G}$ magnetic beads. Then the beads were washed and incubated with Proteinase $\mathrm{K}$ at $55^{\circ} \mathrm{C}$ for $30 \mathrm{~min}$ to digest proteins. The purified RNA was obtained and analyzed by RT-qPCR.

Dual-luciferase reporter assay. In this study, the OIP5-AS1/miRNA interactions were predicted using Starbase (http://starbase.sysu.edu.cn/) and DIANA-LncBase database (http://www.microrna.gr/LncBase). For OIP5-AS1 and miR-340-5p binding activity, OIP5-AS1 fragment containing the binding sites of miR-340-5p, as well as those of the wild-type and mutant sequences were cloned into a pmirGLO Dual-luciferase Vector designated as OIP5-AS1 WT or OIP5-AS1 MUT. For ZEB2 and miR-340-5p binding activity, fragment of 3'UTR ZEB2 containing the binding sites of miR-340-5p, as well as the wild-type and mutant sequences were cloned into a pmirGLO Dual-luciferase Vector designated as ZEB2 WT or ZEB2 MUT. These vectors were co-transfected with either NC mimics or miR-340-5p mimics using Lipofectamine 2000 reagent. At $48 \mathrm{~h}$ after transfection, the relative luciferase activities were recorded by dual-luciferase reporter assay system (Promega) and the values were normalized to the Renilla luciferase activity.

Immunohistochemistry staining and hematoxylin and eosin $(H \& E)$ staining. The lung of nude mice was dissected and fixed in $10 \%$ formaldehyde at room temperature overnight. The embedded samples in paraffin were sectioned into $5 \mu \mathrm{m}$ slices and mounted on glass slides. For immunohistochemical staining, the slides of interest were probed with anti-Ki-67 antibodies (1:500; Abcam) and then the secondary streptavidin-horseradish peroxidase-conjugated antibody staining. Immunoreactivity was visualized by $\mathrm{DAB}$ and lightly counterstained with 5\% hematoxylin. For H\&E staining, slides were deparaffinized and rehydrated in graded ethanol solutions, then in distilled water. After H\&E staining, slides were mounted and examined under a light microscope.

Lentivirus production and in vivo metastasis assay. Full-length cDNA of human OIP5-AS1 was amplified from the mRNA of MCF-7 cells and subcloned into pcDNA3.1 (AddGene). The lentiviral and packaging vectors (AddGene) were co-transfected into HEK293FT cells using Lipofectamine 2000 reagent (ThermoFisher) according to the manufacturer's instructions. Virus was collected and concentrated at $48 \mathrm{~h}$ after transfection.

Twenty healthy 6- to 8-week-old female BALB/c nude mice (The Animal Institute, Jilin University) were used in this study and randomly divided into two groups. The mice were housed in a specific pathogen-free (SPF) facility and exposed to a 12-h light/dark cycle. Water and food were offered ad libitum. After 1 week of acclimatization, MCF-7 cells $\left(1 \times 10^{6}\right)$ infected with LV-OIP5-AS1 or LV-NC were intravenously injected through the tail vein of BALB/c nude mice under isoflurane anaesthesia. After 8 weeks of inoculation, the mice were euthanized and the number of lung metastatic tumors per lung were counted under a dissecting microscope and confirmed by H\&E staining. The experimental protocols were approved by the Animal Care Committee of China-Japan Union Hospital Affiliated to Jilin University.

Statistical analysis. Data are expressed as mean \pm standard error mean (SEM). The Student's t test was employed to compare two groups and one-way ANOVA with post hoc test was used to analyze differences among multiple groups. A value of $\mathrm{P}<0.05$ was considered as statistically significant.

\section{Results}

Interference of OIP5-AS1 represses epithelial-to-mesenchymal transition (EMT) in breast cancer cells by regulating ZEB family proteins. The dysregulation of long non-coding RNA OIP5-AS1 was involved in multiple cancer types associating with overall survival, TNM stage and prognosis $(21,23-25)$. In breast cancer, studies reported that OIP5-AS1 is upregulated in both tumor samples and cell lines (21). We first evaluated the expression levels of OIP5-AS1 in five breast cancer cell lines. The results showed that the relative expression levels of OIP5-AS1 were much higher in the five breast cancer cell lines than in the normal epithelial cell line MCF-10A (Fig. 1A). Then, we chose luminal-type breast cancer cell line MCF-7 and basal B TNBC cell line MDA-MB-231 for further functional studies. To investigate the role of OIP5-AS1 in breast cancer metastasis, we efficiently knocked down OIP5-AS1 with siRNAs in MCF-7 and MDA-MB-231 cell lines (Fig. 1B) and analyzed the cell migration and invasion properties. In the wound healing assay, siOIP5-AS1 groups showed a slower migration rate than the siNC group (Fig. 1C). Furthermore, knockdown of OIP5-AS1 in the two cell lines significantly inhibited cell invasion (Fig. 1D). Next, we assessed the effects of the downregulation of OIP5-AS1 on the expression of epithelial-to-mesenchymal transition (EMT) markers. The protein analysis results indicated that the epithelial marker E-cadherin was increased whereas the mesenchymal markers $\mathrm{N}$-cadherin and Vimentin were decreased (Fig. 1E). These results suggested that downregulation of OIP5-AS1 repressed epithelial-to-mesenchymal transition (EMT). Considering the importance of transcription factors in EMT co-ordination, we further tested the expression of EMT-related transcription factors (ZEB1, ZEB2, Snail, Slug and Twist). In the siOIP5-AS1 group, ZEB1 and ZEB2 were significantly downregulated whereas the expression of Snail, Slug and Twist were not affected (Fig. 1F). Thus, we speculated that OIP5-AS1 may exert functions in EMT through regulating ZEB family proteins.

OIP5-AS1 directly targeted miR-340-5p. LncRNAs exert function in various aspects of cellular function and biological process in either nucleus or cytoplasm. In nucleus, lncRNAs may take part in chromatin remodeling and modification or gene expression prior to transcription, whereas IncRNAs in cytoplasm mainly participate in post-transcriptional 

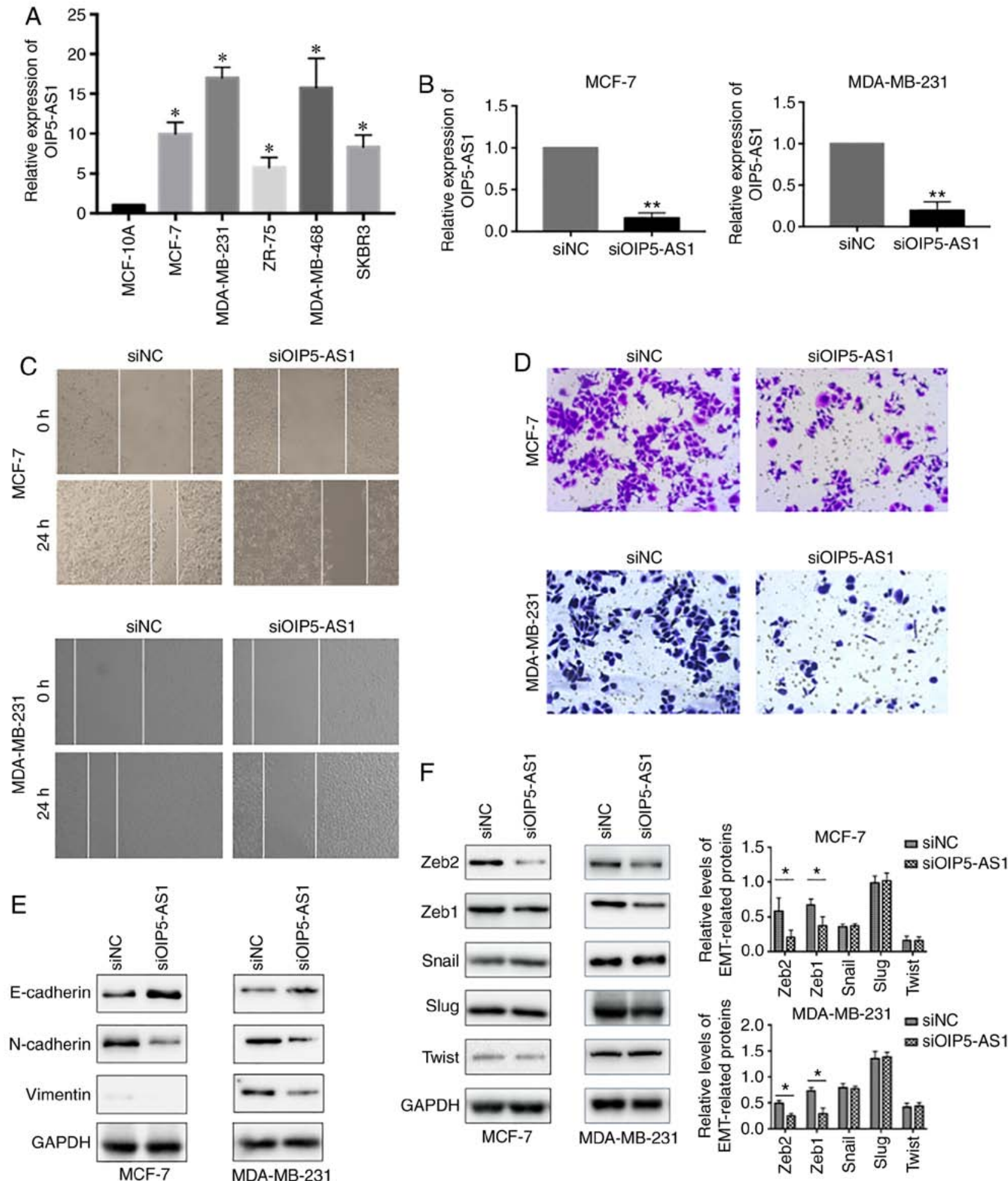

Figure 1. Downregulation of OIP5-AS1 repressed migration, invasion and epithelial-to-mesenchymal transition in breast cancer cells. (A) RT-qPCR analysis of OIP5-AS1 expression in breast cancer cell lines compared with normal mammary MCF-10A epithelial cells. " $\mathrm{P}<0.05$. (B) RT-qPCR analysis of OIP5-AS1 expression with siNC or siOIP5-AS1 transfection in MCF-7 and MDA-MB-231 cells. ${ }^{* *} \mathrm{P}<0.01$ compared with siNC. (C) Migration of MCF-7 and MDA-MB-231 cells in siNC or siOIP5-AS1 transfection groups was determined by wound healing assay. siOIP5-AS1 resulted at a slower closing rate. (D) Transwell invasion assay demonstrated less invaded cells with siOIP5-AS1 transfection in MCF-7 and MDA-MB-231 cells. (E) The protein expression of EMT markers (E-cadherin, N-cadherin and vimentin) indicated the suppression of epithelial-to-mesenchymal transition with siOIP5-AS1 transfection in MCF-7 and MDA-MB-231 cells. GAPDH was used as an internal control. (F) The protein level of EMT-related transcriptional factors (ZEB2, ZEB1, Snail, Slug and Twist) in siNC or siOIP5-AS1 transfection groups indicating the selective regulation of ZEB2 and ZEB1. GAPDH was used as an internal control. ${ }^{*} \mathrm{P}<0.05$. NC, negative control.

regulation and post-translational modification $(26,27)$. Thus, we assessed subcellular location of OIP5-AS1 by fluorescence in situ hybridization (FISH). The detected OIP5-AS1 was mainly localized in the cytoplasm in MCF-7 cells which indicated OIP5-AS1 may serve as a ceRNA in breast cancer cells (Fig. 2A). 

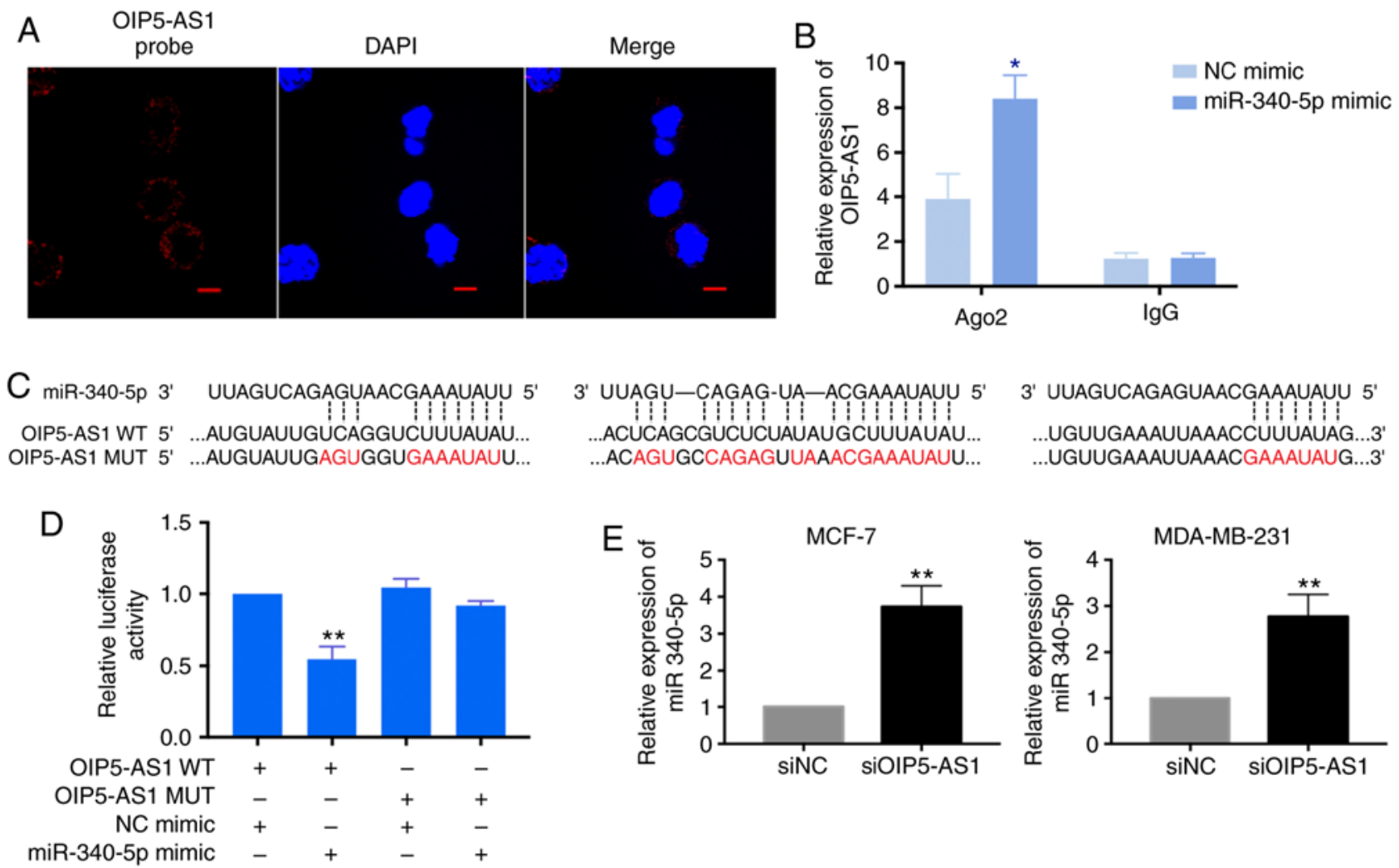

Figure 2. OIP5-AS1 directly targeted miR340-5p. (A) RNA FISH probed endogenous OIP5-AS1 (red) was located in cytoplasm in MCF-7 cells. Scale bar, $10 \mu \mathrm{m}$. (B) RNA-IP analysis confirmed the direct binding of miR-340-5p mimics and OIP5-AS1 in MCF-7 cells. The expression of OIP5-AS1 was detected using RT-qPCR. " $\mathrm{P}<0.05$. (C) The predicted binding sites of miR-340-5p on OIP5-AS1, and target sequences were mutated. (D) Luciferase activity of MCF-7 cells co-transfected with OIP5-AS1 WT or OIP5-AS1 MUT and miR-340-5p mimics or NC mimics. ${ }^{* *} \mathrm{P}<0.01$ compared with OIP5-AS1 WT and NC mimics group. (E) Stem-loop RT-qPCR analysis of miR-340-5p with siNC or siOIP5-AS1 transfection in MCF-7 and MDA-MB-231 cells. ** P<0.01 compared with siNC group.

In this study, the OIP5-AS1/miRNA interactions were predicted using Starbase and DIANA tools. Among the predicted miRNAs, miR-340-5p possesses three target sites on OIP5-AS1. In order to confirm that miR-340-5p is the target gene of OIP5-AS1, we performed anti-Ago2 RIP assay and dual luciferase reporter assay. In anti-Ago2 RIP assay, the endogenous OIP5-AS1 was specifically enriched in miR-340-5p mimics-transfected cells when compared with NC mimics group (Fig. 2B). We constructed the OIP5-AS1 wild-type and mutant reporter plasmids according to the binding sequences of miR-340-5p (Fig. 2C). The dual luciferase reporter assay showed that the reduced luciferase activity was only found in the miR-340-5p mimics and OIP5-AS1 wild-type co-transfection groups but not in the OIP5-AS1 mutant co-transfection group (Fig. 2D). Moreover, we tested the expression of miR-340-5p with OIP5-AS1 knockdown in breast cancer cells. After two days with siOIP5-AS1 transfection, the level of miR-340-5p was increased in MCF-7 and MDA-MB-231 cells (Fig. 2E). Taken together, these results confirmed the direct binding activity between OIP5-AS1 and miR-340-5p in breast cancer cells.

miR-340-5p is downregulated in breast cancer cells and regulates $Z E B 2$ expression. A recent study reported that miR-340-5p was negatively associated with distant metastasis in invasive breast cancers (28). Thus, we measured the relative expression of miR-340-5p in breast cancer cell lines. The level of miR-340-5p was decreased in MCF-7, MDA-MB-231, ZR-75, MDA-MB-468 and SKBR3 cells as compared to human breast epithelial cell line MCF10A (Fig. 3A).

Next, we screened mRNA targets of miR-340-5p using TargetScan and Starbase tools and found the 3'UTR of ZEB2 mRNA contains two binding sites for miR-340-5p. We transfected miR-340-5p mimics into MCF-7 cells and detected the expression of ZEB2 mRNA by RT-qPCR. With the miR-340-5p overexpression (Fig. 3B), the level of ZEB2 mRNA was decreased (Fig. 3C). We further confirmed the direct binding between ZEB2 mRNA and miR-340-5p by dual luciferase reporter assay. The ZEB2 3'UTR was constructed and the mutant form was designed according to the miR-340-5p binding sequences (Fig. 3D). As shown in Fig. 3E, the luciferase activity was only reduced in the ZEB2 3'UTR wild-type and miR-340-5p mimics co-transfection group which suggested the direct binding between ZEB2 mRNA and miR-340-5p. In addition, we examined the effects of miR-340-5p on the protein expression of ZEB2 by overexpression of either miR-340-5p mimics or inhibitors in MCF-7 cells. Similarly, the level of ZEB2 was decreased with miR-340-5p mimics transfection whereas it was increased with miR-340-5p inhibitors transfection (Fig. 3F and G). Collectively, these results supported that miR-340-5p regulates ZEB2 expression by binding to complementary sequences in the 3'UTR of ZEB2 mRNA. 

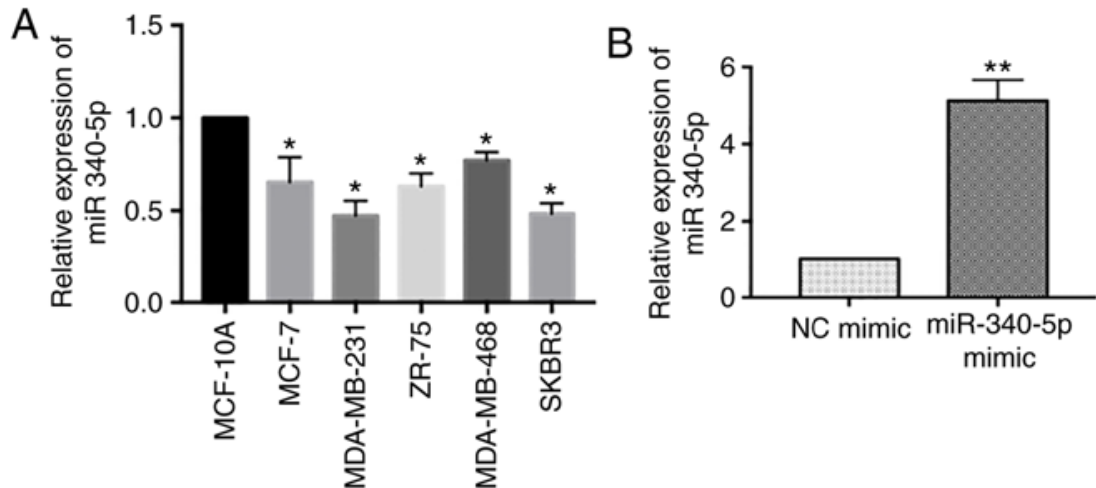

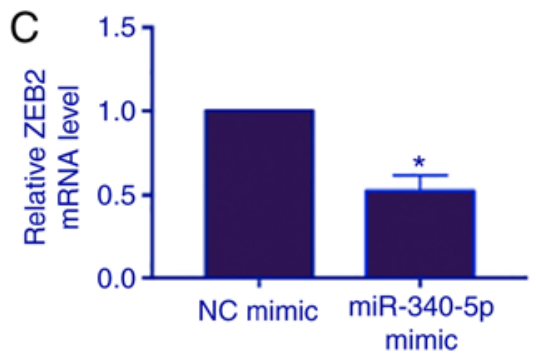

$\mathrm{E}$

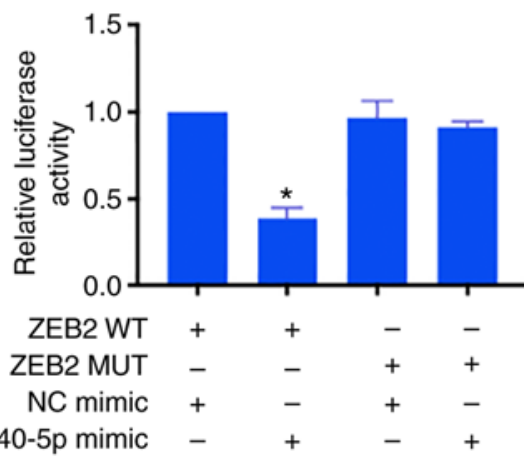

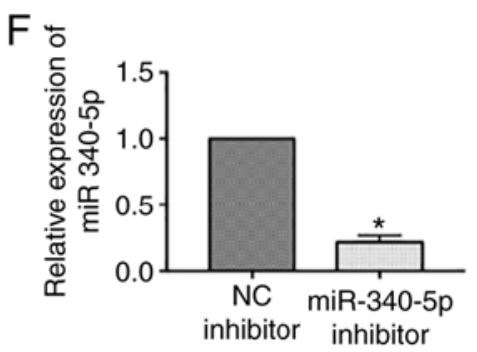
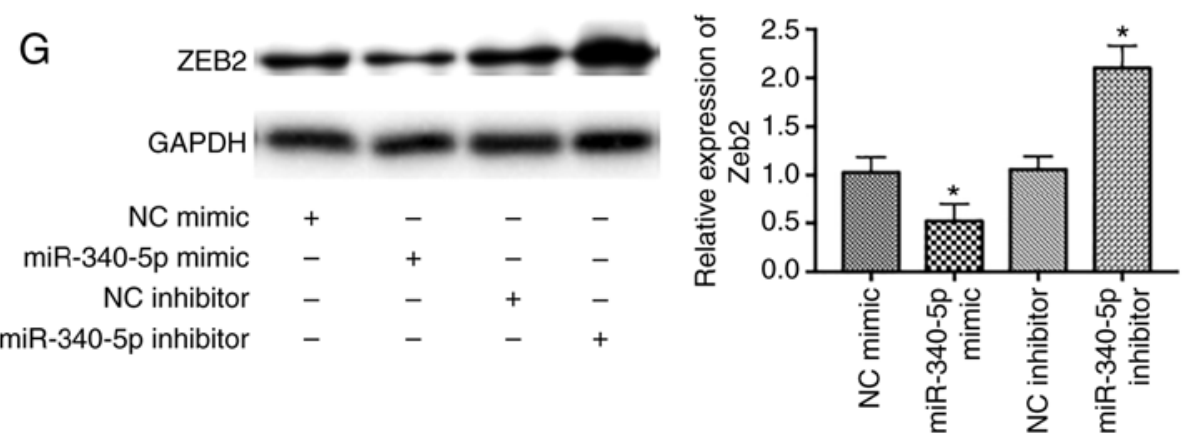

Figure 3. miR-340-5p is downregulated in breast cancer cells and regulates ZEB2 expression. (A) RT-qPCR analysis of miR-340-5p expression in breast cancer cell lines compared with normal mammary MCF-10A epithelial cells. ${ }^{*} \mathrm{P}<0.05$. (B) The relative expression of miR-340-5p in MCF-7 cells transfected with miR-340-5p mimics. ** $\mathrm{P}<0.01$ compared with NC mimics group. (C) RT-qPCR analysis of relative ZEB2 mRNA expression in MCF-7 cells transfected with miR-340-5p mimics. "P<0.05 compared with NC mimics group. (D) The predicted binding sites of miR-340-5p on 3'UTR of ZEB2 and the target sequences were mutated. (E) Luciferase activity of MCF-7 cells co-transfected with ZEB2-WT or ZEB2-MUT and miR-340-5p mimics or NC mimics. ${ }^{*} \mathrm{P}<0.05$ compared with ZEB2-WT and NC mimics group. (F) The relative expression of miR-340-5p in MCF-7 cells transfected with miR-340-5p inhibitors. "P<0.05 compared with NC inhibitor group. (G) The protein level of ZEB2 in MCF-7 cells. miR-340-5p mimics repressed ZEB2 expression and miR-340-5p inhibitors increased ZEB2 expression. ${ }^{\mathrm{P}}<0.05$.

OIP5-AS1 regulates ZEB2 indirectly through sponging $m i R-340-5 p$. We next explored whether OIP5-AS1 regulates ZEB2 expression through sponging miR-340-5p. The ZEB2 mRNA expression was decreased with either knockdown of OIP5-AS1 or overexpression of miR-340-5p mimics; however, this effect was reversed by miR-340-5p inhibitors (Fig. 4A). Then, we tested the protein level of ZEB2. The miR-340-5p inhibitors also reversed the repressed effect of OIP5-AS1 knockdown and miR-340-5p inhibitors alone upregulated ZEB2 expression. ZEB2 is a known transcriptional repressor of E-cadherin. In this experiment, we found that the protein level of E-cadherin was inversely correlated with the ZEB2 level (Fig. 4B). Moreover, we examined the cell invasion ability. The siOIP5-AS1 group showed a decreased number of invasive cells which was reversed by miR-340-5p inhibitors. miR-340-5p inhibitors alone enhanced invasive ability. However, knockdown of ZEB2 markedly repressed cell invasion even with miR-340-5p inhibitors, suggesting that ZEB2 is a downstream factor (Fig. 4C and D). Overall, these results demonstrated that OIP5-AS1 regulates ZEB2 indirectly through sponging miR-340-5p.

OIP5-AS1 promotes breast cancer cells into lung metastasis in vivo. To determine whether OIP5-AS1 causes breast cancer cell metastasis in vivo, the metastasis assay was conducted and the primary pulmonary metastasis was observed. We overexpressed OIP5-AS1 by lentivirus infection in MCF-7 cells and then injected cells into nude mice via tail vein (Fig. 5A). The protein level of ZEB2 was elevated by OIP5-AS1 overexpression (Fig. 5B). The LV-OIP5-AS1 group showed 


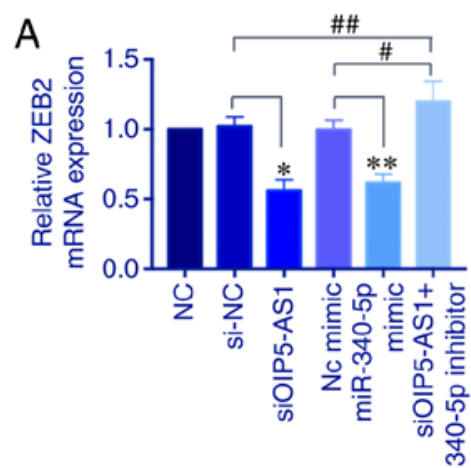

B
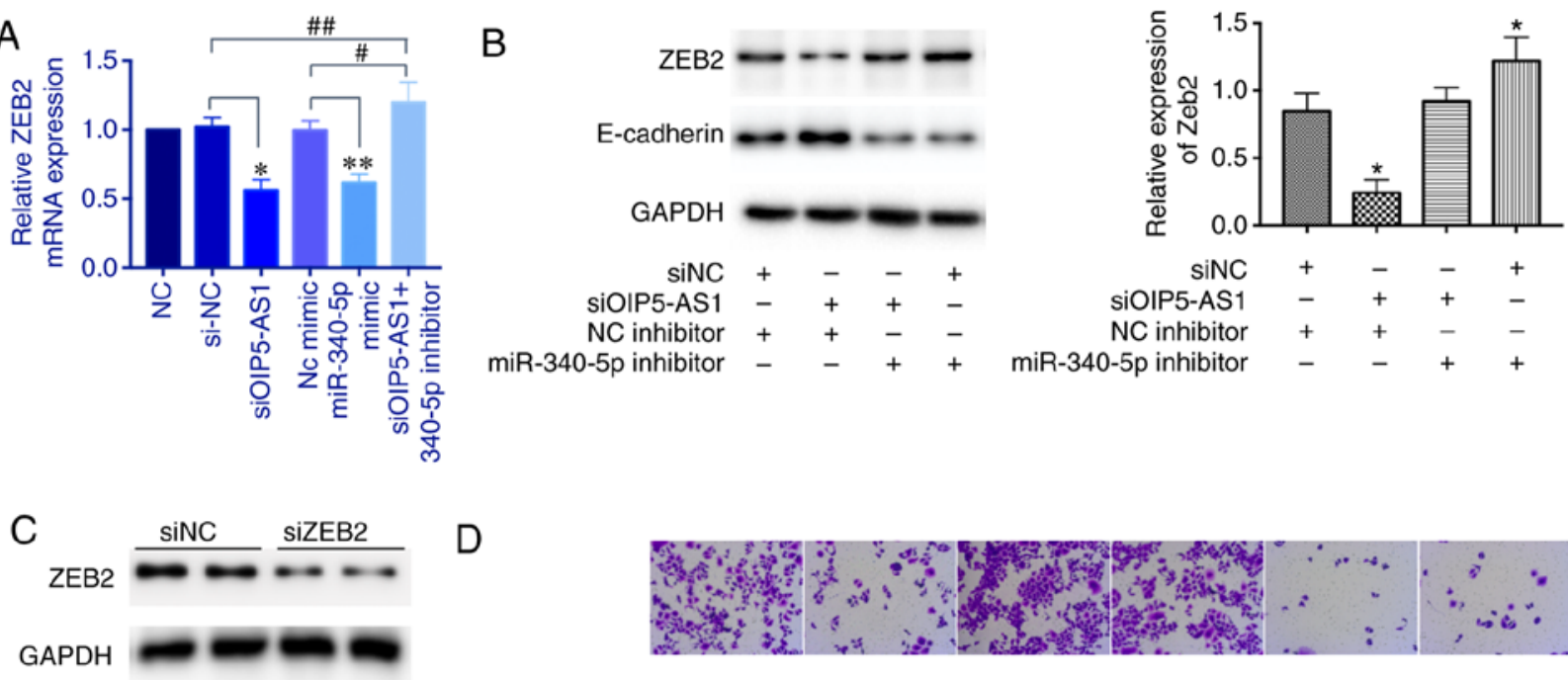

$\begin{array}{rllllll}\text { siNC } & + & - & + & - & - & - \\ \text { SiOIP5-AS1 } & - & + & - & + & - & - \\ \text { NC inhibitor } & + & + & - & - & + & - \\ \text { miR-340-5p inhibitor } & - & - & + & + & - & + \\ \text { siZEB2 } & - & - & - & - & + & +\end{array}$

Figure 4. OIP5-AS1 regulated ZEB2 indirectly through sponging miR-340-5p. (A) RT-qPCR analysis of relative ZEB2 mRNA expression in MCF-7 cells. Either siOIP5-AS1 or miR-340-5p mimics repressed the ZEB2 expression. ${ }^{*} \mathrm{P}<0.05,{ }^{\# \#} \mathrm{P}<0.05$ vs. siNC; ${ }^{* *} \mathrm{P}<0.05,{ }^{\#} \mathrm{P}<0.05$ vs. NC mimics. (B) The protein level of ZEB2 and E-cadherin in MCF-7 cells transfected with siNC or siOIP5-AS1 and NC inhibitors or miR-340-5p inhibitors. "P<0.05. (C) The protein level of ZEB2 in MCF-7 cells transfected with siNC or siZEB2. (D) Transwell invasion assay demonstrated cell invasion in different groups. Less invaded cells were shown with siOIP5-AS1 transfection but rescued by miR-340-5p inhibitor.
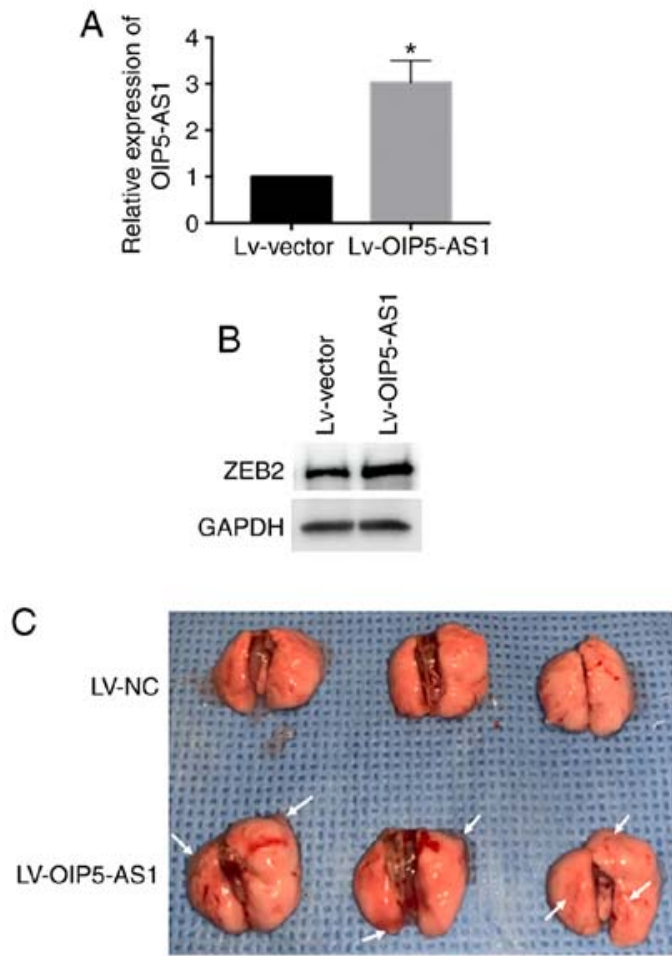

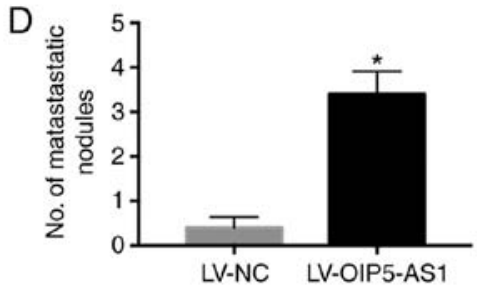

$\mathrm{E}$ LV-NC

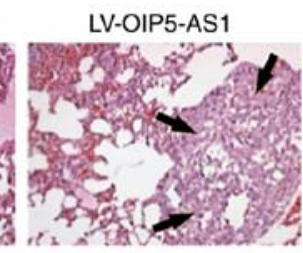

$\mathrm{F}$

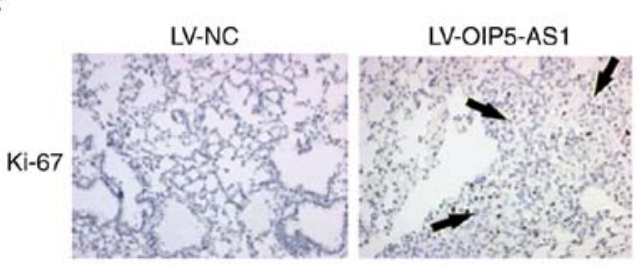

Figure 5. OIP5-AS1 promoted breast cancer cells into lung metastasis in vivo. (A) RT-qPCR analysis of OIP5-AS1 expression in Lv-vector and Lv-OIP5-AS1 infection cells. ${ }^{*} \mathrm{P}<0.05$. (B) The expression of ZEB2 proteins in Lv-vector and Lv-OIP5-AS1 infection cells. (C) The lung tissues of nude mice in Lv-NC and Lv-OIP5-AS1 groups. (D) The number of metastatic lung nodules. ${ }^{*} \mathrm{P}<0.05$. (E) H\&E staining of metastatic lung nodules in Lv-NC group and Lv-OIP5-AS1 group. (F) Ki-67 staining of metastatic lung nodules in Lv-NC group and Lv-OIP5-AS1 group. Arrows indicate metastatic nodules.

marked lung colonization and increased metastatic lung nodules compared with the LV-NC group (Fig. 5C and D). We performed H\&E staining of the metastatic lung tissue in the LV-OIP5-AS1 group. The results were consistent with our observation showing increased metastatic lung nodules (Fig. 5E). Furthermore, the metastatic tumors were positively stained with $\mathrm{Ki}-67$ the marker of cell proliferation (Fig. 5F). In conclusion, these results demonstrate that overexpression 
of OIP5-AS1 promotes breast cancer cells into lung metastasis in vivo.

\section{Discussion}

In the present study, we investigated the role of long non-coding RNA OIP5-AS1 in breast cancer metastasis. We found that OIP5-AS1 was upregulated in five breast cancer cell lines which was consistent with earlier studies and in agreement with supporting evidence from genome-wide analysis of human cancers indicating the prevalent upregulation of OIP5-AS1 $(21,29)$. In vivo experiments also confirmed the effects of OIP5-AS1 in breast cancer cells on lung metastasis. Furthermore, knockdown of OIP5-AS1 markedly weakened cell migration and invasion abilities and inhibited epithelial-to-mesenchymal transition (EMT). These results suggest the pivotal role of OIP5-AS1 in breast cancer metastasis and indicate its potential to be a marker for metastatic breast cancer or for therapeutic evaluation. Moreover, we provided evidence that ZEB2 is an important effector of OIP5-AS1 dysregulation and this association was evident through the regulation of miR-340-5p.

Emerging evidence reveals the role of long non-coding RNAs (LncRNAs) in tumorigenesis and tumor metastasis as the regulator for key gene expression at either transcriptional or translational levels (30). Studies interfered metastasis-associated lncRNAs, such as MALAT1, NEAT1 and BCAR4, showed significant metastasis inhibition $(14,31,32)$. OIP5-AS1 is a newly identified lncRNA, the dysregulation of which has been found in multiple cancer types including breast cancer (33). It is involved in cancer cell proliferation showing a G2/M to G0/G1-phase arrest. Silencing of OIP5-AS1 has been shown to inhibit cell proliferation in multiple cancers $(20,21,23,24)$. In addition, downregulation of OIP5-AS1 has been shown to regulate EMT markers E-cadherin and to reduce metastasis in lung adenocarcinoma (23). Similar results were also obtained in hepatoblastoma demonstrating the involvement of OIP5-AS1 in EMT progress (34). Together with our findings, the functions of OIP5-AS1 in cancer metastasis have been verified in multiple cancer types. Thus, further investigations are needed to validate the network of OIP5-AS1 with clinical stages in related cancer types. In our study, we only examined the function of OIP5-AS1 in the regulation of EMT-related proteins in MCF-7 and MDA-MB-231 cells. These two breast cancer cell lines represent different molecular subtypes of breast cancer which show different metastasis capabilities. Although the regulation of EMT-related proteins was confirmed in these two cell lines, more experiments should be performed in multiple subtypes of breast cancer cell lines due to the different metastatic ability and diversity in the molecular interactions involved even in the same cancer type. Moreover, the general upregulation of OIP5-AS1 has been revealed in different cell lines, but the varying expression values that correlate to metastatic ability is not clear.

OIP5-AS1 was probed using FISH assay was predominantly in the cytoplasm which indicates the potential role of being ceRNAs. Findings have shown that lncRNAs act as ceRNAs which compete for miRNAs to regulate the expression of target genes (12). In the present study, we tested miR-340-5p according to the predicted binding sequences from TargetScan and Starbase tools. The results of RIP assay and dual luciferase reporter assay demonstrated the direct binding of OIP5-AS1 and
miR-340-5p. Interestingly, it has been reported that miR-340-5p is negatively associated with distant metastasis in invasive breast cancers, which suggests the pivotal role of miR-340-5p in metastasis (28). Our results elucidate the ability of miR-340-5p to target ZEB2 which is a new finding confirmed by regulation at both the mRNA and protein levels. Long non-coding RNAs have the potential binding ability with multiple miRNAs through complementary sequences. Several miRNAs were reported to target OIP5-AS1 in the literature, such as miR-129-5p, miR-448, miR-378a-3p and miR-498 (21,23,35,36). Notably, in our results, the siOIP5-AS1-suppressed EMT process in breast cancer cells was markedly blocked by miR-340-5p inhibitors, suggesting a specific inhibitory role of miR-340-5p for OIP5-AS1 in metastasis process. However, the experiments validating the OIP5-AS1/miR-129-5p/ZEB2 axis was only performed in MCF-7 cells which is a potential limitation of this study. Therefore, this molecular mechanism needs to be confirmed in other breast cancer cell lines. Additionally, the functions of long non-coding RNA as miRNA sponge allow us to consider its regulatory networks in tumor biology. More genome-wide analysis and follow-up functional studies on OIP5-AS1 should carried out to understand its diverse role in different types of cancer.

In conclusion, we identified the OIP5-AS1/miR-3405p/ZEB2 axis in breast cancer cell metastasis. OIP5-AS1 facilitated breast cancer metastasis by sponging miR-340-5p to upregulate ZEB2 mRNA transcripts. The current results provide a new direction for the further investigation of molecular mechanism of breast cancer metastasis. Defining the underlying mechanisms of differentially expressed lncRNA in cancers may be useful in developing novel strategies for cancer diagnosis and treatment.

\section{Acknowledgements}

Not applicable.

\section{Funding}

No funding was received.

\section{Availability of data and materials}

All data generated or analyzed during the present study are included in this published article.

\section{Authors' contributions}

LM and HL conceived and designed the study. LM, XY and DZ performed the experiments. LM and HL wrote the manuscript. All authors read and approved the final manuscript and agreed to be accountable for all aspects of the work in ensuring that questions related to the accuracy or integrity of any part of the work are appropriately investigated and resolved.

\section{Ethics approval and consent to participate}

Animal experiments conducted in the present study were approved by Animal Care Committee of China-Japan Union Hospital Affiliated to Jilin University. 


\section{Patient consent for publication}

Not applicable.

\section{Competing interests}

The authors declare that they have no competing interests.

\section{References}

1. Li N, Deng Y, Zhou L, Tian T, Yang S, Wu Y, Zheng Y, Zhai Z, Hao Q, Song D, et al: Global burden of breast cancer and attributable risk factors in 195 countries and territories, from 1990 to 2017: Results from the global burden of disease study 2017. J Hematol Oncol 12: 140, 2019.

2. DeSantis CE, Ma J, Sauer AG, Newman LA and Jemal A: Breast cancer statistics, 2017, racial disparity in mortality by state. CA Cancer J Clin 67: 439-448, 2017.

3. Cardoso F, Costa A, Senkus E, Aapro M, André F, Barrios CH, Bergh J, Bhattacharyya G, Biganzoli L, Cardoso MJ, et al: 3rd ESO-ESMO international consensus guidelines for advanced breast cancer (ABC 3). Ann Oncol 28: 16-33, 2017.

4. Malhotra GK, Zhao X, Band H and Band V: Histological, molecular and functional subtypes of breast cancers. Cancer Biol Ther 10: 955-960, 2010.

5. Xiao W, Zheng S, Yang A, Zhang X, Zou Y, Tang H and Xie X: Breast cancer subtypes and the risk of distant metastasis at initial diagnosis: A population-based study. Cancer Manag Res 10 5329-5338, 2018

6. Kozłowski J, Kozłowska A and Kocki J: Breast cancer metastasis-insight into selected molecular mechanisms of the phenomenon. Postepy Hig Med Dosw (Online) 69: 447-451, 2015

7. Chaffer CL, Juan BPS, Lim E and Weinberg RA: EMT, cell plasticity and metastasis. Cancer Metastasis Rev 35: 645-654, 2016.

8. Peinado H, Olmeda D and Cano A: Snail, Zeb and bHLH factors in tumour progression: An alliance against the epithelial phenotype? Nat Rev Cancer 7: 415-428, 2007.

9. Lee JY, Park MK, Park JH, Lee HJ, Shin DH, Kang Y, Lee CH and Kong G: Loss of the polycomb protein Mel-18 enhances the epithelial-mesenchymal transition by ZEB1 and ZEB2 expression through the downregulation of miR-205 in breast cancer. Oncogene 33: 1325-1335, 2014.

10. Evans JR, Feng FY and Chinnaiyan AM: The bright side of dark matter: lncRNAs in cancer. J Clin Invest 126: 2775-2782, 2016.

11. Calle AS, Kawamura Y, Yamamoto Y, Takeshita F and Ochiya T: Emerging roles of long non-coding RNA in cancer. Cancer Sci 109: 2093-2100, 2018

12. Marchese FP, Raimondi I and Huarte M: The multidimensional mechanisms of long noncoding RNA function. Genome Biol 18: 206, 2017.

13. Gupta RA, Shah N, Wang KC, Kim J, Horlings HM, Wong DJ, Tsai MC, Hung T, Argani P, Rinn JL, et al: Long non-coding RNA HOTAIR reprograms chromatin state to promote cancer metastasis. Nature 464: 1071-1076, 2010.

14. Mendell JT: Targeting a long noncoding RNA in breast cancer. N Engl J Med 374: 2287-2289, 2016.

15. Li W, Zhai L, Wang H, Liu C, Zhang J, Chen W and Wei Q: Downregulation of LncRNA GAS5 causes trastuzumab resistance in breast cancer. Oncotarget 7: 27778-27786, 2016

16. Huang YS, Chang CC, Lee SS, Jou YS and Shih HM: Xist reduction in breast cancer upregulates AKT phosphorylation via HDAC3-mediated repression of PHLPP1 expression. Oncotarget 7: 43256-43266, 2016

17. Tian T, Wang M, Lin S, Guo Y, Dai Z, Liu K, Yang P, Dai C, Zhu Y, Zheng Y, et al: The impact of lncRNA dysregulation on clinicopathology and survival of breast cancer: A systematic review and meta-analysis. Mol Ther Nucleic Acids 12: 359-369, 2018.

18. Ulitsky I, Shkumatava A, Jan CH, Sive H and Bartel DP: Conserved function of lincRNAs in vertebrate embryonic development despite rapid sequence evolution. Cell 147: 1537-1550, 2011.

19. Meseure D, Alsibai KD, Nicolas A, Bieche I and Morillon A: Long noncoding RNAs as new architects in cancer epigenetics, prognostic biomarkers, and potential therapeutic targets. BioMed Res Int 2015: 320214, 2015.
20. Naemura M, Kuroki M, Tsunoda T, Arikawa N, Sawata Y, Shirasawa $\mathrm{S}$ and Kotake Y: The long noncoding RNA OIP5-AS1 is involved in the regulation of cell proliferation. Anticancer Res 38: 77-81, 2018.

21. Zeng H, Wang J, Chen T, Zhang K, Chen J, Wang L, Li H, Tuluhong D, Li J and Wang S: Downregulation of long non-coding RNA Opa interacting protein 5-antisense RNA 1 inhibits breast cancer progression by targeting sex-determining region Y-box 2 by microRNA-129-5p upregulation. Cancer Sci 110: 289-302, 2019.

22. Livak KJ and Schmittgen TD: Analysis of relative gene expression data using real-time quantitative PCR and the 2(-Delta Delta C(T)) method. Methods 25: 402-408, 2001.

23. Deng J, Deng H, Liu C, Liang Y and Wang S: Long non-coding RNA OIP5-AS1 functions as an oncogene in lung adenocarcinoma through targeting miR-448/Bcl-2. Biomed Pharmacother 98: 102-110, 2018.

24. Wang Y, Shi F, Xia Y and Zhao H: LncRNA OIP5-AS1 predicts poor prognosis and regulates cell proliferation and apoptosis in bladder cancer. J Cell Biochem Nov 18, 2018 (Epub ahead of print).

25. Ren X, He J, Qi L, Li S, Zhang C, Duan Z, Wang W, Tu C and Li Z: Prognostic and clinicopathologic significance of long non-coding RNA opa-interacting protein 5-antisense RNA 1 in multiple human cancers. Artif Cells Nanomed Biotechnol 48: 353-361, 2020.

26. Batista PJ and Chang HY: Long noncoding RNAs: Cellular address codes in development and disease. Cell 152: 1298-1307, 2013.

27. Chen LL: Linking long noncoding RNA localization and function. Trends Biochem Sci 41: 761-772, 2016.

28. Rohan TE, Wang T, Weinmann S, Wang Y, Lin J, Ginsberg M and Loudig O: A miRNA expression signature in breast tumor tissue is associated with risk of distant metastasis. Cancer Res 79: 1705-1713, 2019.

29. Arunkumar G, Anand S, Raksha P, Dhamodharan S, Rao HPS, Subbiah S, Murugan AK and Munirajan AK: LncRNA OIP5-AS1 is overexpressed in undifferentiated oral tumors and integrated analysis identifies as a downstream effector of stemness-associated transcription factors. Sci Rep 8: 7018, 2018.

30. Jiang MC, Ni JJ, Cui WY, Wang BY and Zhuo W: Emerging roles of lncRNA in cancer and therapeutic opportunities. Am J Cancer Res 9: 1354-1366, 2019.

31. Arun G, Diermeier S, Akerman M, Chang KC, Wilkinson JE, Hearn S, Kim Y, MacLeod AR, Krainer AR, Norton L, et al: Differentiation of mammary tumors and reduction in metastasis upon Malat1 lncRNA loss. Genes Dev 30: 34-51, 2016.

32. Xing Z, Lin A, Li C, Liang K, Wang S, Liu Y, Park PK, Qin L, Wei Y, Hawke DH, et al: IncRNA directs cooperative epigenetic regulation downstream of chemokine signals. Cell 159: 1110-1125, 2014.

33. Comijn J, Berx G, Vermassen P, Verschueren K, van Grunsven L, Bruyneel E, Mareel M, Huylebroeck D and van Roy F: The two-handed $\mathrm{E}$ box binding zinc finger protein SIP1 downregulates E-cadherin and induces invasion. Mol Cell 7: 1267-1278, 2001.

34. Zhang Z, Liu F, Yang F and Liu Y: Kockdown of OIP5-AS1 expression inhibits proliferation, metastasis and EMT progress in hepatoblastoma cells through up-regulating miR-186a-5p and down-regulating ZEB1. Biomed Pharmacother 101: 14-23, 2018.

35. Wang M, Sun X, Yang Y and Jiao W: Long non-coding RNA OIP5-AS1 promotes proliferation of lung cancer cells and leads to poor prognosis by targeting miR-378a-3p. Thorac Cancer 9: 939-949, 2018

36. Zhang X, Xu X, Ge G, Zang X, Shao M, Zou S, Zhang Y, Mao Z, Zhang J, Mao F, et al: miR-498 inhibits the growth and metastasis of liver cancer by targeting ZEB2. Oncol Rep 41: 1638-1648, 2019.

This work is licensed under a Creative Commons Attribution-NonCommercial-NoDerivatives 4.0 International (CC BY-NC-ND 4.0) License. 